\title{
La cohésion dans les productions écrites d'étudiants en anglais de spécialité : un problème culturel?
}

John Osborne

\section{(2) OpenEdition}

\section{Journals}

Édition électronique

URL : http://journals.openedition.org/asp/4090

DOI : 10.4000/asp.4090

ISSN : 2108-6354

Éditeur

Groupe d'étude et de recherche en anglais de spécialité

\section{Édition imprimée}

Date de publication : 1 décembre 1994

Pagination : 205-216

ISSN : 1246-8185

\section{Référence électronique}

John Osborne, «La cohésion dans les productions écrites d'étudiants en anglais de spécialité : un problème culturel ? », ASp [En ligne], 5-6 | 1994, mis en ligne le 18 décembre 2013, consulté le 19 avril 2019. URL : http://journals.openedition.org/asp/4090 ; DOI : 10.4000/asp.4090

Ce document a été généré automatiquement le 19 avril 2019

Tous droits réservés 


\title{
La cohésion dans les productions écrites d'étudiants en anglais de spécialité : un problème culturel ?
}

\author{
John Osborne
}

\section{Introduction}

1 Les productions écrites d'apprenants avancés, même lorsqu'elles contiennent peu d'erreurs lexicales ou morpho-syntaxiques, trahissent souvent leurs origines par des déviances stylistiques, dont l'une des principales sources semble être une maitrise imparfaite des phénomènes de cohésion. Les étudiants en anglais de spécialité, évidemment, ne sont pas à l'abri de ce problème ; le court extrait suivant, par son excès de cohésion, en donne un aperçu :

(1) Nevertheless, Nike is doing well financially. On the contrary, Adidas has strong problems, and LA Gear too. However, Reebok is very powerful on this market.

Mais on peut se demander, plus généralement, si les difficultés que peuvent rencontrer nos étudiants dans la production d'un texte qu'un lecteur anglophone jugerait comme normalement cohésif sont d'ordre culturel. Sont-elles, du moins en partie, le résultat d'habitudes de pensée, d'ordonnancement des idées ou de stratégie discursive qui seraient particuliers à une langue-culture (française en l'occurrence)?

Depuis le célèbre article de Kaplan (1966), l'idée qu'il peut exister différents cultural thought patterns a été largement discutée et affinée, notamment par Kaplan lui-même :

In fact, it is now my opinion that all of the various rhetorical modes [...] are possible in any language - i.e., in any language which has written text. The issue is that each language has certain clear preferences, so that while all forms are possible, all forms do not occur with equal frequency or in parallel distribution. (1987:10)

Pour d'autres, si l'existence de différences culturelles n'est pas en cause, c'est plutôt une maitrise imparfaite des marqueurs explicites qui serait source de confusion dans la communication écrite; il s'agirait donc d'un problème essentiellement linguistique et non 
pas linguistico-culturel. Tyler \& Bro par exemple $(1992,1993)$ montrent que des jugements de cohérence portés par des lecteurs natifs sont influencés davantage par la (mauvaise) utilisation de marqueurs discursifs (discourse structuring miscues) que par l'organisation des idées.

5 Pour d'autres encore, ce sont les idées elles-mêmes qui seraient souvent à l'origine du problème. L'utilisation abusive de marqueurs de cohésion serait alors la trace d'une confusion mentale, lorsque l'étudiant, quelles que soient sa langue et sa culture d'origine, tente d'imposer une logique de surface sur un texte où les idées sont mal maîtrisées :

There is even some evidence that an increase in cohesive ties in a text signals an area of difficulty which the (poor) writer tries to overcome by the abundance of superficial links. The writer almost attempts to yoke the resistant ideas together by force. (Crew 1990 : 321)

6 Cette dernière hypothèse paraît difficile à vérifier (comment mesurer la résistance des idées ?), mais elle peut troubler l'interprétation des données; nous y reviendrons à la fin de cet article. Dans l'immédiat, il convient de définir la portée - modeste - de cette étude.

\section{Description de l'étude}

7 La mobilité actuelle des étudiants européens nous offre dans un même groupe - et donc accomplissant exactement les mêmes tâches - des apprenants d'origines linguistiques et culturelles diverses, et la possibilité de comparer ainsi des productions de même nature. Pour cette étude, nous avons choisi un groupe d'étudiants en maitrise de Langues étrangères appliquées, groupe composé de 51 francophones et de 16 non-francophones (allemands, hongrois et danois), et avons analysé toutes leurs productions écrites pendant un semestre.

8 Parallèlement, afin d'avoir un autre point de comparaison, la même analyse a été effectuée sur les productions d'un groupe de l'année inférieure, en licence LEA, composé exclusivement de francophones (ce groupe comportait quelques non-francophones, mais leurs productions n'ont pas été prises en compte).

9 Les étudiants travaillaient sur des études de cas en commerce/gestion, tirées, pour la maîtrise, de David (1993) et, pour la licence, de Casler \& Palmer (1989). Les productions analysées, individuelles ou, plus souvent, collectives, étaient des contributions à l'analyse des cas, et présentaient des réponses argumentées à des questions comme «Should Nike narrow its product line in athletic shoes?", "How can Nike maintain a competitive advantage over LA Gear and Reebok? », etc. Le tableau 1 résume la composition du corpus.

Tableau 1 : composition du corpus

\begin{tabular}{|l|l|}
\hline Groupe 1 (étudiants de licence, francophones) & 32244 mots \\
\hline Groupe 2 (étudiants de maîtrise, francophones) & 72287 mots \\
\hline Groupe 3 (étudiants de maîtrise, non-francophones) & 30832 mots \\
\hline Total & 135363 mots \\
\hline
\end{tabular}


La comparaison, pour cette première étape de l'étude, est purement quantitative, et porte uniquement sur l'aspect le plus visible de la cohésion: l'utilisation des connecteurs. Seules sont comptabilisées les liaisons inter-phrastiques; les opérateurs de subordination, même placés en tête de phrase, ne sont pas comptés.

\section{Résultats}

11 La première mesure - la fréquence d'utilisation, tous connecteurs confondus - est très grossière, mais montre déjà un écart intéressant entre les étudiants francophones et nonfrancophones, alors que le nombre de connecteurs utilisés par les deux groupes francophones ( 1 et 2 ) est à peu près constant :

Tableau 2 : nombre de connecteurs utilisés

\begin{tabular}{|l|l|}
\hline & connecteurs $/ 1000$ mots \\
\hline Groupe 1 & 12,37 \\
\hline Groupe 2 & 12,58 \\
\hline Groupe 3 & 8,23 \\
\hline
\end{tabular}

12 Des différences plus fines apparaissent lorsque l'on fait l'inventaire des connecteurs les plus utilisés. Les tableaux 3 à 5 montrent, pour chacun des groupes, les douze connecteurs les plus fréquents ( $\mathrm{f}=$ nombre d'occurrences pour 1000 mots).

La comparaison des groupes 1 et 2 fait apparaître une distribution un peu plus régulière des connecteurs; pour le groupe 2 , la fréquence globale des connecteurs est légèrement supérieure (voir tableau 2), mais les connecteurs les plus utilisés sont relativement moins fréquents. Ceci ne semble pas être dû, comme on pourrait le penser, à un répertoire potentiel plus étendu, car c'est le groupe 1 qui utilise le plus grand nombre de connecteurs différents.

Tableau 3 : connecteurs les plus fréquents (groupe 1 )

\begin{tabular}{|l|l|l|}
\hline rang & mot de liaison & f. \\
\hline 1 & So & 1,64 \\
\hline 2 & But & 1,24 \\
\hline 3 & Moreover & 0,96 \\
\hline 4 & And & 0,78 \\
\hline 5 & However & 0,68 \\
\hline 6 & Therefore & 0,62 \\
\hline
\end{tabular}




\begin{tabular}{|l|l|c|}
\hline 7 & Indeed & 0,43 \\
\hline 7 & Consequently & 0,43 \\
\hline 7 & On the one hand & 0,43 \\
\hline 10 & Besides & 0,40 \\
\hline 10 & Thus & 0,40 \\
\hline 10 & That is why & 0,40 \\
\hline
\end{tabular}

Tableau 4 : connecteurs les plus fréquents (groupe 2)

\begin{tabular}{|l|l|l|}
\hline rang & mot de liaison & f. \\
\hline 1 & Moreover & 1,13 \\
\hline 2 & Indeed & 1,09 \\
\hline 3 & But & 1,02 \\
\hline 4 & However & 0,77 \\
\hline 5 & So & 0,73 \\
\hline 6 & Therefore & 0,59 \\
\hline 7 & Nevertheless & 0,55 \\
\hline 8 & In fact & 0,43 \\
\hline 8 & Thus & 0,42 \\
\hline 10 & Furthermore & 0,35 \\
\hline 10 & As a matter of fact & 0,35 \\
\hline 12 & That is why & 0,33 \\
\hline
\end{tabular}

14 On constate aussi, entre ces deux groupes francophones, une régression des liaisons les plus simples («So», «And», «But»). Dans le cas de «And» et «But», cette régression s'accompagne d'une promotion de leurs quasi-synonymes, « Moreover » et «However ». Le recul de «So », par contre, ne semble pas profiter à «Therefore » mais, par une évolution que nous commenterons plus loin, plutôt à «Indeed». Dans l'ensemble, les connecteurs les plus prisés par le groupe 2 semblent se distinguer de ceux du groupe 1 , non pas en ce qu'ils traduisent une organisation différente du texte, mais par leur appartenance à un registre un peu plus élevé ou, si l'on préfère, à une culture plus « haute » (cf. Johns 1994). 
Les groupes 2 et 3 ont en commun six connecteurs (" However », "Therefore », «But », " Furthermore », «Thus », « Moreover »), mais sur les deux connecteurs les plus prisés par les francophones, "Moreover» est assez peu utilisé par les non-francophones (avec 6 occurrences pour plus de 30000 mots, il vient en onzième position), et «Indeed » ne l'est pas du tout. Les différences sont donc considérables entre ces deux groupes, et nous verrons qu'elles apparaissent de manière plus systématique lorsqu'on regroupe les connecteurs selon leur fonctionnement.

Tableau 5 : connecteurs les plus fréquents (groupe 3)

\begin{tabular}{|l|l|l|}
\hline rang & mot de liaison & f. \\
\hline 1 & However & 1,14 \\
\hline 2 & Therefore & 0,94 \\
\hline 3 & But & 0,65 \\
\hline 4 & Concerning & 0,52 \\
\hline 5 & Furthermore & 0,45 \\
\hline 5 & Secondly & 0,45 \\
\hline 7 & In addition & 0,42 \\
\hline 8 & Thus & 0,32 \\
\hline 8 & Finally & 0,32 \\
\hline 10 & First of all & 0,29 \\
\hline 11 & Then (énumération) & 0,19 \\
\hline 11 & Moreover & 0,19 \\
\hline
\end{tabular}

16 S'agissant toujours de la hiérarchie individuelle des connecteurs, il peut être intéressant de comparer nos résultats avec ceux obtenus dans une étude de plus grande envergure, dans le cadre de l'International Corpus of Learner English, projet piloté par l'Université Catholique de Louvain (Granger 1993, 1994). Ce corpus informatisé, en cours de constitution, est composé à partir de productions écrites d'apprenants avancés, essentiellement sur des thèmes de société. Mesurer la fréquence d'utilisation des connecteurs n'est évidemment qu'une partie de ce projet, mais les résultats obtenus pour les apprenants francophones sont résumés dans le tableau 6.

17 La comparaison des tableaux 6 et 7 (qui regroupe les résultats pour tous nos étudiants francophones en anglais de spécialité) fait ressortir l'importance du genre des textes analysés. Il n'y a que six connecteurs communs aux deux tableaux (« Moreover », « So », « Indeed », «Therefore », "Thus », «In fact »), dont deux seulement («Indeed », « Thus ») ont des fréquences très proches. Il paraît probable que les écarts (notamment la fréquence de «For example», «For instance», "Of course» dans l'ICLE, alors qu'on ne relève, 
respectivement, que $0,14,0,14$ et 0,10 occurrences pour 1000 mots dans notre corpus) sont dus essentiellement aux différentes argumentations propres à un problème de société et à un problème de gestion.

Tableau 6: connecteurs les plus fréquents, sous-corpus francophone de l'ICLE (d'après Granger 1994)

\begin{tabular}{|l|l|l|}
\hline rang & mot de liaison & f. \\
\hline 1 & In fact & 0,82 \\
\hline 2 & So & 0,78 \\
\hline 3 & Indeed & 0,72 \\
\hline 4 & For example & 0,60 \\
\hline 4 & For instance & 0,60 \\
\hline 6 & Of course & 0,59 \\
\hline 7 & Moreover & 0,58 \\
\hline 8 & However & 0,52 \\
\hline 9 & Thus & 0,40 \\
\hline 10 & On the other hand & 0,39 \\
\hline 11 & On the contrary & 0,38 \\
\hline 12 & Therefore & 0,37 \\
\hline
\end{tabular}

Tableau 7 : connecteurs les plus fréquents, tous étudiants francophones (anglais commerce/ gestion)

\begin{tabular}{|l|l|l|}
\hline rang & mot de liaison & f. \\
\hline 1 & But & 1,09 \\
\hline 2 & Moreover & 1,08 \\
\hline 3 & So & 1,01 \\
\hline 4 & Indeed & 0,79 \\
\hline 4 & However & 0,75 \\
\hline 6 & Therefore & 0,60 \\
\hline 7 & Nevertheless & 0,42 \\
\hline
\end{tabular}




\begin{tabular}{|l|l|c|}
\hline 8 & Thus & 0,41 \\
\hline 9 & In fact & 0,38 \\
\hline 10 & And & 0,36 \\
\hline 11 & That is why & 0,35 \\
\hline 12 & Consequently & 0,33 \\
\hline
\end{tabular}

Il serait toutefois intéressant de savoir s'il y a des phénomènes de cohésion qui, à l'instar de « Thus » et «Indeed » ici, semblent relativement insensibles aux changements de genre. On remarque, par exemple, que malgré une différence considérable d'un corpus à l'autre, « For example » et « For instance » ont, à l'intérieur d'un même corpus, exactement la même fréquence, alors que les anglophones natifs auraient (toujours d'après les relevés de l'ICLE) une nette préférence pour « For example ». Il semblerait donc que « For instance " ait un caractère marqué qui échappe aux apprenants ; l'(in)sensibilité aux préférences d'une langue-culture joue aussi sur le choix des connecteurs.

Les différences d'utilisation de tel ou tel connecteur ne sont pas sans intérêt, mais pour voir apparaître des différences plus systématiques, il est utile de faire des comparaisons par type de connecteur. Cela suppose que l'on ait établi une classification des connecteurs, et on entre alors dans un domaine aux frontières très floues. Halliday \& Hasan (1976) distinguent quatre catégories de conjonction, chacune divisée en 4 souscatégories; Quirk et al. (1985) distinguent sept catégories et huit sous-catégories; Sinclair et al. (1990) en distinguent 6; Souesme (1992) donne huit catégories, dont une " miscellaneous ", pour ne citer que quelques exemples. Lorsque l'on veut rendre compte de l'utilisation des connecteurs par des non-natifs, et même si l'on reclasse les emplois visiblement erronés («At last» pour « Finally», par exemple), il est encore plus malaisé d'éviter l'arbitraire et les catégories « poubelle ». La classification que nous avons adoptée reprend, pour l'essentiel, les quatre grandes catégories de Halliday \& Hasan, avec quelques modifications pour rendre compte du sens particulier qu'ont certains des connecteurs de notre corpus (la liste complète des 67 connecteurs relevés, avec leur classification, est donnée en annexe).

Tableau 8 : fréquence d'utilisation par type de connecteur

\begin{tabular}{|l|l|l|l|}
\hline Connecteurs & Groupe 1 & Groupe 2 & Groupe 3 \\
\hline Addition (additive) & $\mathbf{2 , 9 2}$ & $\mathbf{2 , 8 4}$ & $\mathbf{1 , 4 9}$ \\
\hline ajout & 2,69 & 2,35 & 1,20 \\
\hline illustration & 0,19 & 0,33 & 0,19 \\
\hline reformulation & 0,03 & 0,06 & 0,06 \\
\hline comparaison & $\varnothing$ & 0,10 & 0,03 \\
\hline
\end{tabular}




\begin{tabular}{|l|l|l|l|}
\hline Contraste (adversative) & $\mathbf{3 , 0 4}$ & $\mathbf{3 , 0 4}$ & $\mathbf{2 , 4 3}$ \\
\hline concession & 2,42 & 2,45 & 2,08 \\
\hline opposition & 0,62 & 0,59 & 0,35 \\
\hline Cause (causal) & $\mathbf{4 , 8 3}$ & $\mathbf{4 , 5 7}$ & $\mathbf{1 , 7 8}$ \\
\hline résultat & 4,00 & 2,71 & 1,78 \\
\hline justification & 0,43 & 1,09 & $\varnothing$ \\
\hline explication & 0,40 & 0,77 & $\varnothing$ \\
\hline Organisation (temporal) & $\mathbf{1 , 5 8}$ & $\mathbf{2 , 1 3}$ & $\mathbf{2 , 5 3}$ \\
\hline focalisation & 0,40 & 0,91 & 0,84 \\
\hline énumération & 0,96 & 0,98 & 1,59 \\
\hline conclusion & 0,22 & 0,24 & 0,10 \\
\hline & 12,37 & 12,58 & 8,23 \\
\hline
\end{tabular}

20 Les principales modifications par rapport à la classification de Halliday \& Hasan sont les suivantes. Dans la troisième catégorie (Causal), nous avons ajouté «Indeed», qui n'apparaît pas dans l'inventaire de Halliday \& Hasan, et «In fact »/《 As a matter of fact », qu'ils classent dans leur deuxième catégorie (Adversative). Ce choix est motivé par les détournements de sens de ces connecteurs dans notre corpus, et sera expliqué plus loin. La quatrième catégorie (Temporal) a été élargie et rebaptisée, pour accueillir un ensemble de connecteurs (tels que "Regarding", "Concerning») qui sont fréquents dans notre corpus, et dont le rôle relève d'une fonction de structuration interne semblable à celle des Internal temporals comme « First », « Next », «In conclusion ».

Certaines sous-catégories, enfin, sont empruntées non pas à la classification de Halliday \& Hasan, mais à celle de Quirk et al.

La similitude entre les deux groupes francophones (1 et 2) est beaucoup plus frappante ici. Les fréquences d'utilisation sont remarquablement constantes, si l'on excepte une augmentation des marqueurs de focalisation ("Regarding", "Concerning", etc.) et une tendance à remplacer les liaisons de type p, donc q (marquées par "So ", "Therefore ", etc.) par le lien inverse (q, parce que p, marqué, comme nous le verrons, par « Indeed »).

La comparaison entre les groupes $1+2$ et le groupe 3 fait ressortir plusieurs différences. D'abord, les francophones utilisent deux fois plus de connecteurs d'addition ("Moreover ", etc.) et, par une coïncidence amusante, écrivent des paragraphes deux fois plus courts, au point que si l'on multiplie la longueur moyenne des paragraphes par la fréquence des connecteurs d'addition, le résultat est presque constant (pour le groupe 2 : longueur moyenne d'un paragraphe 33,8 mots $\times 2,84=96$; pour le groupe 3 : longueur moyenne d'un paragraphe 62,7 mots $\times 1,49=93,4)$. 

celle des connecteurs de structuration, grâce en particulier aux énumératifs. Mais les différences les plus marquantes se trouvent dans l'utilisation des liaisons de cause. Non seulement elles sont globalement près de trois fois plus fréquentes chez les francophones, mais certains des connecteurs que nous avons classés dans cette catégorie, et qui sont beaucoup utilisés par les francophones ("Indeed », "In fact », " As a matter of fact ») sont totalement absents du sous-corpus du groupe 3 , mise à part une seule occurrence de «In fact ». On note aussi (voir tableau 6) que «Indeed» et «In fact» sont également très fréquents chez les francophones de l'ICLE. Il est donc intéressant de regarder d'un peu plus près l'utilisation qui est faite de ces connecteurs. En anglais orthodoxe, "Indeed», lorsqu'il assure un lien inter-phrastique, sert généralement à annoncer un degré d'assertion plus grand (cf. Delechelle 1983). Pour les apprenants francophones il semble plutôt annoncer une justification de l'assertion qui précède, comme dans les exemples suivants, tirés de notre corpus :

(2) Another strategic action would be to extend the product line to other sports. Indeed, Nike does not produce all kinds of athletic shoes and that is why it should diversify its range.

(3) Nike cannot consider the European market as an entity. Indeed, Nike has to be aware of the diversity of the cultures, histories and economies that divide the European market.

(4) As far as Reebok is concerned, it is the most dangerous competitor for Nike in Europe. Indeed, if Nike succeeds in keeping the leadership on the American market, Reebok has proved its capacity of innovation and specialization.

L'exemple (2), avec l'enchaînement "Indeed ... that is why ... », est particulièrement clair, mais tous ces exemples contiennent une relation de cause inversée (phrase1/puisque/ phrase2), semblable à celles introduites, en français, par «Car» ou «En effet». Tous pourraient être ré-écrits selon le modèle : phrase2/therefore/phrase1. Nous avons donc classé cet emploi de «Indeed» dans la sous-catégorie que Halliday \& Hasan appellent « reversed causal ». Ils précisent toutefois que cet agencement est peu courant :

The REVERSED form of the causal relation, in which the presupposing sentence expresses the cause, is less usual as a form of cohesion. Within the sentence, it is natural to find the structural expression of cause going in either direction. With the cohesive relation between sentences, however, in which the text unfolds one sentence after another, the logical precedence of cause over effect is reflected in the typical sequence in which sentences related in this way tend to occur. (1976: 257-258)

"Natural », «logical » et «typical » ici trahissent peut être une conception un peu anglocentrique de l'organisation textuelle. Car si la relation causale inversée, marquée notamment par "For ", est effectivement peu habituelle en anglais, il s'agit là d'un phénomène qui ne semble pas aller de soi pour nos apprenants francophones. «Indeed", ou du moins l'utilisation qu'ils en font, paraît bien répondre à leurs habitudes argumentatives, et leur permet, dans les phrases qu'il introduit, d'apporter à la fois une justification et une expansion de l'assertion qui précède.

29 «In fact » et « As a matter of fact » sont classés par Halliday \& Hasan dans la catégorie des adversatifs, mais bien que ces deux connecteurs apparaissent fréquemment dans notre corpus, c'est très rarement avec ce sens contrastif. «In fact » se rencontre parfois dans des relations adversatives, mais sans en être lui-même le pivot ; ce rôle revient à un autre connecteur («But», le plus souvent), tandis que "In fact» sert à étayer le contreargument en y apportant une justification. Le sens contrastif habituel est donc fractionné 
en trois étapes: phrase 1 (assertion),/But/phrase2 (contre-assertion),/In fact/phrase3 (justification), comme dans les exemples qui suivent:

(5) Since Adidas has been present in the market for a long time its executives assert that the consumers will be attracted to the older, more familiar brand. But Nike's quick rise has proved the contrary. In fact, especially among young people, Nike's brand awareness is fairly large.

(6) Nike's profits increased steadily during the early Eighties, but in 1984 Nike lost its dominance. In fact Nike's executives missed a very important market: that for aerobic shoes.

(7) As far as R\&D is concerned, Nike invests only $3 \%$ of profits for innovation. This figure can seem quite low, but we should bear in mind that R\&D in this industry, does not need expensive equipment. In fact most of R\&D is design innovation.

(8) One of Nike's main strength is its deep production line. That enables the brand to offer an incredibly wide choice in the same segment of sportshoes market. However we could wonder about the usefulness of such a broad choice. In fact, Nike sells 300 models of athletic shoes in 900 styles for 20 different sports.

On voit que le rôle de "In fact» se rapproche ici du sens justificatif de «Indeed»; c'est d'ailleurs le sens qu'il semble avoir le plus souvent dans notre corpus, où les phrases introduites par «In fact » fournissent une explication de l'assertion qui précède :

(7) Most of the time, quality equals premium price. The price suggested is then $\$ 40$ or over. It is important to notice that to reduce prices can be dangerous for a company as Nike. In fact this would change its position in people's mind and consequently its prestige would be lost.

(8) Creating and activating a distribution network is generally a long and expensive operation. In fact this operation does not have an immediate effect like an action on price or an advertising campaign.

(9) Very often the wrong choice can affect sales. Selling Nike's shoes in hypermarkets would increase sales in the short term. In fact everyone would like a pair of Nike at a low price. But if we consider the long term view Nike's profits and prestige would be affected.

"As a matter of fact », tel qu'il est employé dans notre corpus, est encore moins adversatif que «In fact ». Ces deux connecteurs, qui en anglais standard ont des significations très proches, ne semblent pas avoir tout à fait le même sens pour nos apprenants francophones. "As a matter of fact ", tout en introduisant une justification de l'assertion qui précède, annonce aussi une explicitation, marquant ainsi un mouvement du général vers le spécifique :

(10) A large amount of budget is invested in communication. As a matter of fact in 1991 Nike spent $\$ 140$ million in advertising in order to position their products as high-performance shoes.

(11) Nike has to be ready to win this market shares war in which it will have to face Reebok and Adidas as its main competitors. As a matter of fact, the three companies are very close to each other.

(12) Concerning its production, Nike has to cope with problems and the brand undergoes a series of criticism concerning its production process based in the new industrialized countries. As a matter of fact, the whole process is subcontracted to South East Asian countries and the firm makes a 100\% margin from the plant to the distributors.

Deux derniers exemples illustreront une association particulièrement intéressante, entre « As a matter of fact » et « Indeed » :

(13) The NBA's aim has been clearly heralded: make profits by turning basketball into the first sport all round the world. As a matter of fact, a lot of things remain to be done on the market in Europe. Indeed, basketball within this continent is not as 
well developed as in the United States where the sportshoes industry is running towards this market segmentation.

(14) For example, there is newly a basketball boom in Europe and especially in France. This wave of basketball fans is drifting into French schools. Indeed, among the young population Michael Jordan and Magic Johnson are becoming more popular than Jean-Pierre Papin and Eric Cantona. As a matter of fact, kids only talk about basketball, only wear basketball clothes and, of course, play basketball. particulière à notre corpus (voir par exemple Connor, 1987); ce qui est plus caractéristique, nous semble-t-il, c'est la façon dont cette organisation apparaît explicitement dans la surface du texte, au besoin par détournement de certains connecteurs. Là se trouve peut être la trace d'habitudes culturelles ou, plus particulièrement, scolaires.

\section{Conclusion}

Lobjectif de cette étude était essentiellement exploratoire : voir si ce genre d'analyse était susceptible de faire apparaître des différences qui mériteraient un examen plus élaboré. Par conséquent, les variables en présence n'ont été contrôlées que de façon assez lâche. Si les tâches demandées aux groupes 2 et 3 étaient identiques, les deux groupes étaient de taille et, vraisemblablement, de composition différentes. La multiplication des échanges entre universités européennes fait que les étudiants qui y participent sont de moins en moins atypiques, mais on ne peut pas exclure la possibilité que certaines des caractéristiques relevées dans les productions de ceux qui composaient 
notre groupe 3 soient simplement le reflet d'une compétence rédactionnelle supérieure à la moyenne.

Cependant, les écarts parfois importants qui apparaissent dans nos résultats bruts semblent indiquer qu'il existe bien des différences dues en partie à des habitudes culturelles, même si ces différences demandent à être confirmées par un meilleur échantillonnage. Au-delà de cette confirmation, d'autres questions intéressantes se posent, dont nous énumérons certaines pour terminer. Dans quelle mesure, d'abord, l'utilisation des connecteurs, telle que nous l'avons observée, jouerait-elle sur des jugements de cohérence portés par des lecteurs natifs ? Parallèlement, y aurait-il des écarts entre les jugements portés par des natifs et ceux des non-natifs (ou entre ceux de non-natifs d'origines culturelles différentes)?

Notre corpus était composé de productions individuelles et collectives; nous n'avons pas cherché à les distinguer, puisque les proportions étaient comparables dans tous les groupes, mais il serait intéressant de savoir comment les conditions de production influent sur les phénomènes de cohésion.

41 Comme nous l'avons vu, l'utilisation des connecteurs peut varier selon le genre des textes analysés; les quelques comparaisons que nous avons pu faire avec d'autres productions de notre groupe 2 semblent confirmer que ces variations subsistent même lorsque les auteurs sont les mêmes. Cependant, il conviendrait de faire des comparaisons sur une plus grande échelle si l'on veut savoir dans quelle mesure tel ou tel phénomène est sensible à un changement de genre, et surtout, s'agissant d'étudiants en anglais de spécialité, si l'on veut distinguer entre les procédés qu'ils adoptent pour parler de leur domaine de spécialité et ceux qui relèvent de leur stratégie générale d'écriture.

Notre étude a porté exclusivement sur les connecteurs ; il est évident que leur utilisation est imbriquée dans un ensemble plus complexe, comportant d'autres mécanismes de cohésion tels que la référence, la substitution, l'ellipse, la cohésion lexicale et la structure thématique. On peut se demander, notamment, si la sur-utilisation de certains connecteurs ne va pas de pair avec une sous-utilisation d'autres procédés, comme cela semble être le cas pour les connecteurs d'addition et la périodicité des paragraphes. Il nous semble, par exemple, que c'est parfois une méconnaissance de la force thématique de «this» qui pousse nos apprenants à avoir recours à des connecteurs bricolés (et souvent inutiles) tels que «Indeed ». Mais cela reste à démontrer.

\section{BIBLIOGRAPHIE}

Casler, K. \& Palmer, D. 1989. Business Assignments. Oxford : Oxford University Press.

Connor, U. 1987. « Argumentative patterns in student essays: cross cultural differences ». In Connor, U. \& R. Kaplan (dir.), Writing Across Languages: Analysis of L2 Text. Reading, MA : Adison Wesley.

Crew, W.J. 1990. « The illogic of logical connectives ». ELT Journal 44/4, 316-325.

David, F. 1993. Strategic Management. New York : Macmillan. 
Delechelle, G. 1983. « 'En effet' et ses traductions en anglais ».Tréma 8, 49-67.

Granger, S. 1993. «The International corpus of learner English ». In Conference on English Language Research on Computerized Corpora. Amsterdam \& Atlanta : Rodopi.

Granger, S. 1994. « The learner corpus: a revolution in applied linguistics ». English Today.

Halliday, M.A.K. \& Hasan, R. 1976. Cohesion in English. Londres : Longman.

Johns, A. « LSP and culture: A special relationship ». ASp 5-6,11-19.

Kaplan, R. 1966. « Cultural thought patterns in intercultural education ». Language Learning, 16, $1-20$.

Kaplan, R. 1987. « Cultural thought patterns revisited ». In Connor, U. \& R. Kaplan (dir.), Writing Across Languages: Analysis of L2 Text. Reading, MA : Adison Wesley.

Quirk, R., Greenbaum, S., Leech, G., Svartvik, J. 1985. A Comprehensive Grammar of the English Language. Londres : Longman.

Sinclair, J. et al. 1990. Cobuild English Grammar. Londres : Collins.

Souesme, J-C. 1992. Grammaire anglaise en contexte. Paris : Ophrys.

Tyler, A. \& Bro, J. 1992. «Discourse structure in non-native English discourse: the effect of ordering and interpretive cues on perceptions of comprehensibility ». Studies in Second Language Acquisition 14/1, 71-86.

Tyler, A. \& Bro, J. 1993. « Discourse processing effort and perceptions of comprehensibility in non-native discourse: the effect of ordering and interpretive cues revisited ». Studies in Second Language Acquisition 15/4, 505-522.

\section{ANNEXES}

\section{Annexe: classification des connecteurs}

\begin{tabular}{|l|l|l|}
\hline Addition & Contraste & \\
(additive)* & (adversative) & Organisation \\
ajout & concession & focalisation \\
reinforcing* & concessive & \\
\hline
\end{tabular}




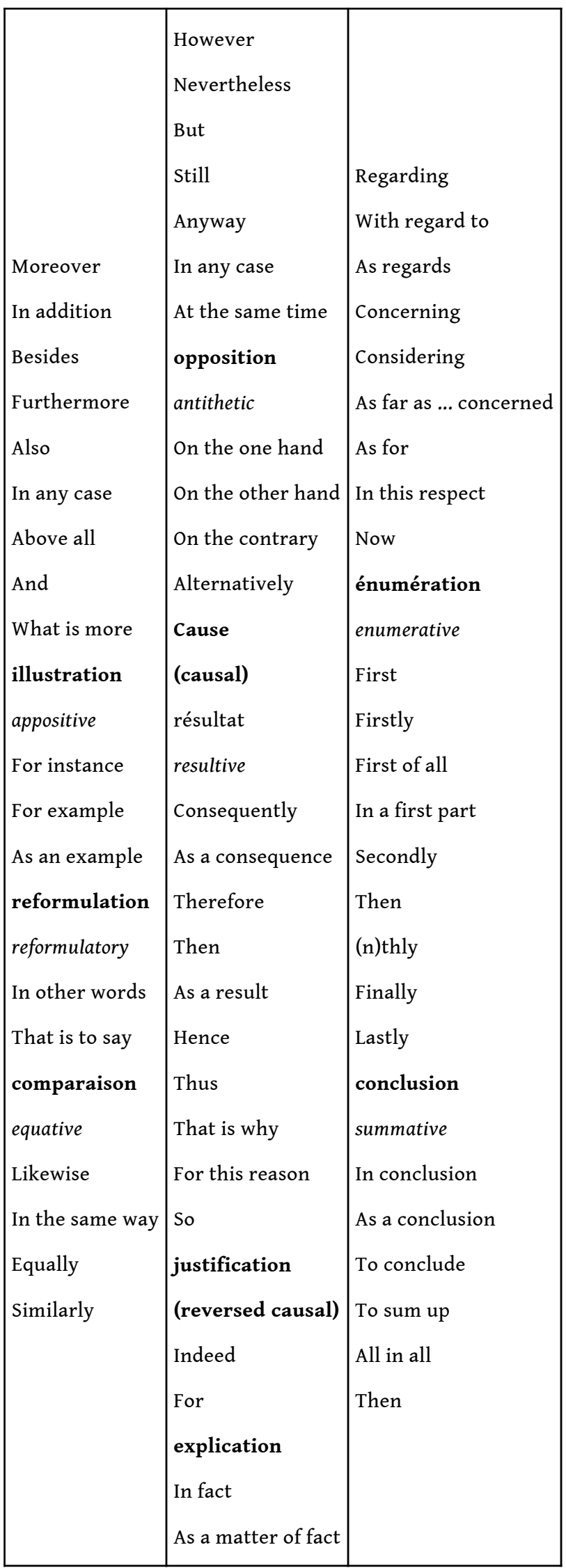


* Les termes entre parenthèses correspondent à la classification de Halliday \& Hasan ; ceux en italiques correspondent à la classification de Quirk et al.

\section{RÉSUMÉS}

Cette étude compare la fréquence d'utilisation de connecteurs inter-phrastiques dans les productions écrites d'apprenants francophones et non-francophones en anglais de spécialité (LEA Commerce international). La comparaison fait apparaitre des différences parfois importantes, notamment dans l'utilisation de connecteurs d'addition et de justification. Ces écarts, et le détournement de certains connecteurs (Indeed, In fact) par les Francophones, semblent refléter des différences culturelles dans l'élaboration et la rédaction d'un texte.

This study compares the frequency of inter-sentential connecters in the writing of French and non-French students of ESP (English with Business Studies). The comparison shows sometimes quite considerable differences, particularly in the use of additive and reversed-causal connecters. It is suggested that these discrepancies, and the idiosyncratic use of certain connecters ("Indeed", "In fact") by French-speaking learners, may reflect cultural differences in the preparation and writing of texts.

\section{INDEX}

Mots-clés : cohésion, connecteur, production écrite

Keywords : cohesion, connector, writing

\section{AUTEUR}

\section{JOHN OSBORNE}

John Osborne enseigne à l'Université de Savoie. John.Osborne@univ-savoie.fr 